\title{
Pomegranate transplant stress can be ameliorated by Rhizophagus intraradices under nursery management
}

\author{
María Josefina Bompadre ${ }^{1 *}$, Roxana Paula Colombo ${ }^{1}$, Vanesa Analía Silvani ${ }^{1}$, Laura \\ Fernández Bidondo ${ }^{1}$, Alejandro Guillermo Pardo ${ }^{2}$, Juan Antonio Ocampo ${ }^{3}$, Alicia Margarita \\ Godeas $^{1}$
}

${ }^{1}$ Departamento de Biodiversidad y Biología Experimental, Facultad de Ciencias Exactas y Naturales, Universidad de Buenos Aires, Intendente Güiraldes 2160, Ciudad Universitaria, 4to piso, Pabellón 2, C1428EGA, Buenos Aires, Argentina. ${ }^{2}$ Laboratorio de Micología Molecular, Departamento de Ciencia y Tecnología, Universidad Nacional de Quilmes, Roque Sáenz Peña 352, B1876BXD, Bernal, Buenos Aires, Argentina. ${ }^{3}$ Departamento de Microbiología del Suelo y Sistemas Simbióticos, Estación Experimental del Zaidín, CSIC, Profesor Albareda, 1, 18008, Granada, Spain. *Corresponding author; maria.bompadre@unq.edu.ar

\begin{abstract}
Arbuscular mycorrhizal fungi (AMF) establish an obligate mutualistic symbiosis with many plant species, increasing the uptake of phosphorous and other low-mobile nutrients by roots. In addition, AMF improve biotic and abiotic stress tolerance of host plants. Under these conditions, reactive oxygen species (ROS) increase occasional damage to proteins, lipids and DNA. Antioxidative enzymes in plants can play an important role in detoxifying ROS, thereby alleviating oxidative stress. In nursery practices, plants are subjected at least to two transplant conditions before being transplanted outside. It is important to achieve an optimal plant size to withstand environmental or other stresses when plants are transplanted into the field. The transplantation process can be considered a stress because plants have to adapt to new abiotic and biotic (rhizospheric) conditions. The aim of this study was to evaluate the effect of two Rhizophagus intraradices (N.C. Schenck \& G.S. Sm.) C. Walker \& A. Schüßler strains, GA5 and GC2, single and co-inoculated under two-transplant soil conditions, sterile and non-sterile, using cuttings of pomegranate (Punica granatum L.) as a model plant. These results showed that the GA5 single strain-inoculated plants improved growth and antioxidative enzyme responses to two transplant stress conditions. In conclusion, early mycorrhizal inoculation generates healthy plants that are more protected against environmental conditions, thereby improving plant transplant stress tolerance.
\end{abstract}

Keywords: Antioxidative enzymes, arbuscular mycorrhizal fungi, nursery production, ROS, transplant. 


\section{Introduction}

Agricultural practices, particularly under nursery conditions, make use of large amounts of chemical fertilizers, but only a fraction of them are effectively used by plants (Marin, 2005). Some plants have developed adaptive mechanisms to better scavenge soil phosphorus, such as enhancing root system development by increasing the number and elongation of radical roots hairs, acidification of rhizosphere-solubilizing inorganic phosphorus and association with arbuscular mycorrhizal fungi (AMF) (Marin, 2005).

AMF establish an obligate mutualistic symbiosis with many plant species by increasing the uptake of phosphorous and other low-mobile nutrients via roots. Its importance is due to the role in the maintenance of soil and plant community structure and diversity. Other benefits include the improvement of biotic and abiotic stress resistance of host plants (Ruiz-Lozano, 2003). Modern techniques of plant propagation include the use of AMF inoculants, such as bioprotectors, phytostimulators or biofertilizers, during nursery production, but co-occurring AMF strains can differ in root colonization ability, effects on plant performance and compatibility (Helgason et al. 2002; Janoušková et al., 2009). However, interspecific interactions between AMF may also result in negative feedback in plant growth (Violi, et al., 2007).

In nursery practices, plants are subjected at least to two transplant conditions before being transplanted outside. The horticultural process is generally misunderstood and results in the loss of many plants (Koller, 1977); thus, it is important to achieve an optimal plant size to withstand environmental or other stresses when plants are transplanted into the field (Liptay et al., 1998). The beneficial effects provided by AMF can be divided into conditions within and outside of the nursery. Under nursery conditions, beneficial effects can be less evident due to controlled conditions.
It is likely that AMF do not accelerate the culture process within the nursery but promote outside transplantation success (Marin, 2005). The transplantation process can be considered an abiotic stress because plants have to adapt to a new rhizospheric condition. Moreover, a homeostatic imbalance in reactive oxygen species (ROS) caused by different stress conditions in plants can result in the oxidative destruction of cells (Mittler et al., 2004). Plants can scavenge ROS via antioxidant enzymes. In addition, AMF associations can help plants to alleviate oxidative stress by enhancing the activities of antioxidant enzymes, such as catalase (CAT), superoxide dismutase (SOD), ascorbate peroxidase (APX), and other factors (Alguacil et al., 2003; Wu et al., 2010), but the response of individual enzymes has been shown to vary depending on the fungal species and host plant (RuizLozano et al., 2012).

Pomegranate (Punica granatum L.) is a small deciduous tree of the family Lythraceae that reaches $2-5 \mathrm{~m}$ in height. Its propagation is mediated by cuttings and has a first stage of cultivation in a nursery, which includes a rooting period and two instances of pot rustication. Under this condition, plants are sensitive to dehydration after transplantation and a lack of fertilizer mainly during flowering (Parodi 1978; Aseri et al., 2008; Franck 2009; Khattab et al., 2011). Munkvold et al. (2004) reported that the ability of AMF to promote plant growth could vary greatly among strains of the same symbiont species. The aim of this study was to evaluate the protective effect of two strains of Rhizophagus intraradices (N.C. Schenck \& G.S. Sm.) C. Walker \& A. Schüßler with different colonization strategies observed in vitro and ex vitro, single inoculated and co-inoculated, on pomegranate (Punica granatum L.) performance under sterile and non-sterile transplantation conditions. 


\section{Materials and Methods}

\subsection{Plants}

The experiment was carried out in a South Temperate Zone. A vigorous and youthful mother plant of Punica granatum L. cv. Plena Voss $3 \mathrm{~m}$ in height was chosen from the Departamento de Producción Vegetal (Facultad de Agronomía, FAUBA, Argentina; -34.593794, -58.48586) for the study. A total of 300 branch cuttings of $7 \mathrm{~cm}$ in length and two original shoots from the same pomegranate plant were used, to eliminate genetic variability (Figure 1). Indole butyric acid
$(2,500 \mathrm{ppm})$ was added to a wound made at the bottom of each cutting (Knight et al., 2005). Rooting was performed on a raised table with perlite at a density of 1,000 cuttings $\mathrm{m}^{-2}$ with intermittent irrigation (MIST) to maintain a constant humidity, reduce temperature and create appropriate microclimate conditions for cuttings rooting. The raised table was divided into four parts separated with plastic panels to prevent the advancement of mycorrhizae and roots of each treatment applied (described below). A $70 \%$ rooting success was obtained after 40 days of cultivation (Figure 1).

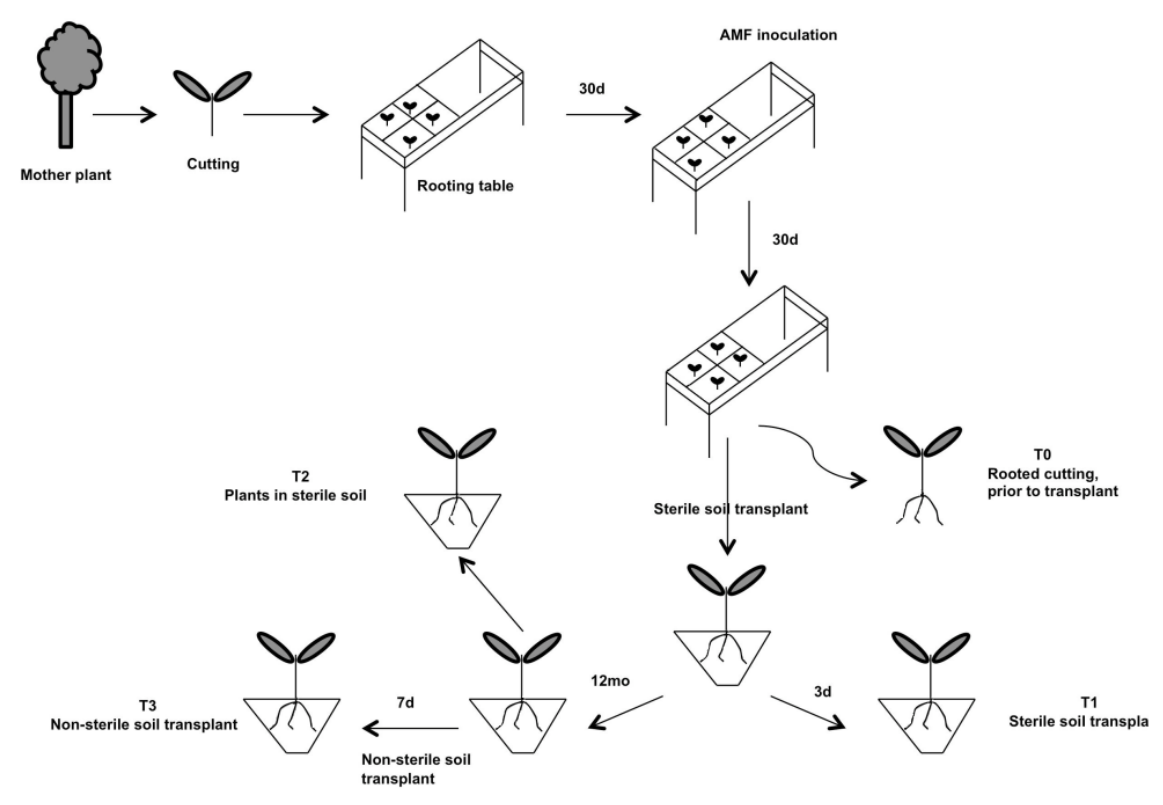

Figure 1. Experimental design diagram 


\subsection{Mycorrhizal inocula}

Two strains (GA5 and GC2) of $R$. intraradices were provided by the Banco de Glomeromycota In Vitro (BGIV). Both strains were selected by their different colonization strategies in vitro and ex vitro tested in our laboratory (Silvani et al., 2014). The strains were propagated on Trifolium repens $\mathrm{L}$. as the host plant in $1.5 \mathrm{~L}$ plastic pots with perlite: soil mixture $(3: 1, \mathrm{v}: \mathrm{v})$ sterilized by tyndallization $\left(100{ }^{\circ} \mathrm{C}\right.$ for $1 \mathrm{~h}$, three consecutive days). The soil characteristics were $\mathrm{pH}$ of $7.1,12.08 \mathrm{~g} \mathrm{~kg}^{-1}$ total $\mathrm{C}, 1.1 \mathrm{~g} \mathrm{~kg}^{-1} \mathrm{~N}, 34.2 \mathrm{mg} \mathrm{kg}^{-1} \mathrm{P}$, $0.9 \mathrm{cmol} \mathrm{kg}^{-1} \mathrm{~K}, 7.5 \mathrm{cmol} \mathrm{kg}^{-1} \mathrm{Ca}, 1.7 \mathrm{cmol} \mathrm{kg}^{-1} \mathrm{Mg}$, and $0.2 \mathrm{cmol} \mathrm{kg}^{-1} \mathrm{Na}$. All plants were maintained for 4 months under greenhouse conditions $(450 \mu \mathrm{mol}$ protons $\mathrm{m}^{-2} \mathrm{~s}^{-2}, 400-700 \mathrm{~nm} ; 16 \mathrm{~h}$ light $-8 \mathrm{~h}$ dark; $25^{\circ} \mathrm{C}$ day and $18{ }^{\circ} \mathrm{C}$ night temperatures; $60-70 \%$ relative humidity). Plants were watered with Hewitt (1952) solution without phosphorous every 15 days and unwatered thereafter until dry to obtain dry mycorrhizal inoculum.

\subsection{Experimental design}

After 30 days of rooting, we created 3-cm-deep furrows between groups of cuttings, and added $10 \mathrm{~g}$ of dry inoculum (see mycorrhizal inocula section) of the strain used in each treatment (inoculation with $R$. intraradices strain: GA5; inoculation with $R$. intraradices strain: $\mathrm{GC} 2$; inoculation of a 1:1 mixture of both strains: GA5+GC2; and control without AMF: C). The control treatment received $10 \mathrm{~g}$ of autoclaved inoculum supplemented with a filtrate $(<20 \mu \mathrm{m})$ of mycorrhizal inoculum (to provide microbial populations) (Figure 1). After 30 days of inoculation on a raised rooting table under nursery conditions, 48 cuttings were kept in the rooting table (T0), 48 cuttings were transplanted to $0.5 \mathrm{~L}$ plastic pots with a mixture of tyndallized perlite: vermiculite: soil mixture (2:1:1; v:v:v) for $3 \mathrm{~d}(\mathrm{~T} 1)$, and 96 cuttings were transplanted into $1.5 \mathrm{~L}$ plastic pots with the same mixture, and grown for a period of 12 months under nursery conditions (Figure 1). They were maintained at $70 \%$ of field capacity and were irrigated with Hewitt (1952) solution without phosphorous addition every month. Following, 48 plants were transplanted into $5 \mathrm{~L}$ plastic pots with non-sterile soil and irrigated with water only immediately at transplantation. They were maintained for 7 days under nursery conditions (T3). The other 48 plants were kept in $1.5 \mathrm{~L}$ plastic pots in sterile-soil (T2) (Figure 1). Thus, the following samplings were made: (T0) Cuttings rooted in raised table (cuttings were at field capacity), (T1) 3 days after transplant to plastic pots with tyndallized substrate. Initially, pots were set up at field capacity (70 ( $1.8 \%)$, and $\mathrm{T} 1$ pots were found at 63.6 ( $0.9 \%$ of field capacity. Plants were placed in $1.5 \mathrm{~L}$ plastic pots with sterile soil and maintained 12 months at 70 ( $1.6 \%$ of field capacity (T2). Next, after 7 days, the places were transplanted from $5 \mathrm{~L}$ plastic pots to non-sterile soil (T3). Plants immediately transplanted to non-sterile soil were found at 40.1 ( $1.6 \%$ of field capacity; after 7 days, they reached values of 24.8 ( $0.8 \%$ of field capacity at harvest. Plants were not fertilized at those samplings. The field capacity was calculated by weighing the substrate before and after drying at $105{ }^{\circ} \mathrm{C}$ for $24 \mathrm{~h}$ (dry substrate $* 100 \%$ / fresh substrate).

\subsection{Growing parameters}

Mycorrhization was tested 30 days after inoculation (T0) for the sterile transplant condition, and before the non-sterile transplant condition (T2). The proportion of mycorrhizae (MI\%), arbuscules (A\%), and vesicles $(\mathrm{V} \%)$ in each stained sample were determined independently (McGonigle et al., 1990). Plant survival percentage, fresh and dry weights and water content of shoots and roots were quantified. The shoot-to-root biomass ratio was evaluated. The 
mycorrhizal dependency percentage (MD\%) was measured as described in Menge et al. (1978).

\subsection{Enzymes and lipid peroxidation}

For enzymatic measurements, fresh plant materials of $P$. granatum L. were weighed and $1 \mathrm{~g}$ of shoots and of roots from each plant was immediately frozen in liquid nitrogen to maintain the integrity of the tissue. Each sample was pulverized in a mortar, with polyvinylpolypyrrolidone (PVPP, $0.06 \mathrm{~g}$ per $6 \mathrm{ml}^{-1}$ extraction buffer) and $5 \mathrm{ml}$ of extraction buffer $(\mathrm{KH}-$ ${ }_{2} \mathrm{PO}_{4}-\mathrm{K}_{2} \mathrm{HPO}_{4} 50 \mathrm{mmol} \mathrm{L}^{-1}, \mathrm{pH} 7.8$, plus $0.1 \mathrm{mmol} \mathrm{L}^{-1}$ EDTA). The resulting mixture was filtered through a nylon membrane to remove cell debris and centrifuged at 20,000 $\mathrm{g}$ for 20 minutes. Supernatants were aliquoted and maintained at -70 (C until further use.

The intracellular activities of the following enzymes were measured: catalase (CAT) was based on the decrease in absorbance at $240 \mathrm{~nm}$ caused by the disappearance of $\mathrm{H}_{2} \mathrm{O}_{2}$ (Aebi, 1984), superoxide dismutase (SOD) was measured at $560 \mathrm{~nm}$ according to its capacity to inhibit the photochemical reduction of nitroblue tetrazolium in the presence of riboflavin (Beyer and Fridovich, 1987) and ascorbate peroxidase (APX) was measured as the absorbance at $290 \mathrm{~nm}$ while ascorbic acid was oxidized (Hossain and Asada, 1984). Enzymatic extracts from the shoots and roots were used to measure the malondialdehyde (MDA) content after the reaction with a solution of trichloroacetic acid and thiobarbituric acid as previously described by Hodges et al. (1999). Enzyme activities and MDA content measurements were standardized by protein determination (PROT) (Bradford, 1976).

\subsection{Statistical analysis}

The experiments listed consisted of a randomized block factorial design with two factors: 1) AMF strains inoculation and 2) transplant condition. All data were subjected to a two-way analysis of variance (ANOVA) followed by the Tukey test (honest significant difference HSD) $(\mathrm{P}<0.05)$. Assumptions of homogeneity of variance and normality were confirmed (Clewer and Scarisbrick, 2001). Statistical procedures were performed using the software STATISTICA 6.0.

\section{Results}

Among the different treatments, there were no differences regarding mycorrhization percentages, proportion of arbuscules and vesicles (data not shown). Single inoculated cuttings showed significantly less survival percentage $(75.00 \pm 6.67 \%$ in GA5 and $73.30 \pm 7.59 \%$ in GC2) compared to control plants $(93.92 \pm 1.29 \%)$; no differences were observed in co-inoculated plants $(77.54 \pm 6.50 \%)$. Shoot fresh weight significantly decreased in control plants and co-inoculated plants under transplantation (Figure 2a). Shoot biomass was significantly higher in coinoculated plants at $\mathrm{T} 0$, but at $\mathrm{T} 1$, they decreased to similar values in comparison to the control treatment (Figure 2b). Shoot water content decreased for all treatments at $\mathrm{T} 1$, but it was significantly marked in control plants (Figure 2c). In roots at T1, a significant increase in fresh weight was observed in GA5inoculated plants compared to control plants at T0 (Figure 2e). However, no differences were observed in root biomass (Figure 2f). The water content was higher for all treatments at $\mathrm{T} 1$ and significantly higher in GA5-inoculated plants compared to control plants at T0 (Figure 2g). However, inoculated plants decreased shoot and root MD at transplant (Figure 2d, h). Co-inoculated roots had less MD at T1 condition than a single inoculation (Figure $2 \mathrm{~h}$ ). No differences were observed in the shoot: root biomass ratio (data not shown). 

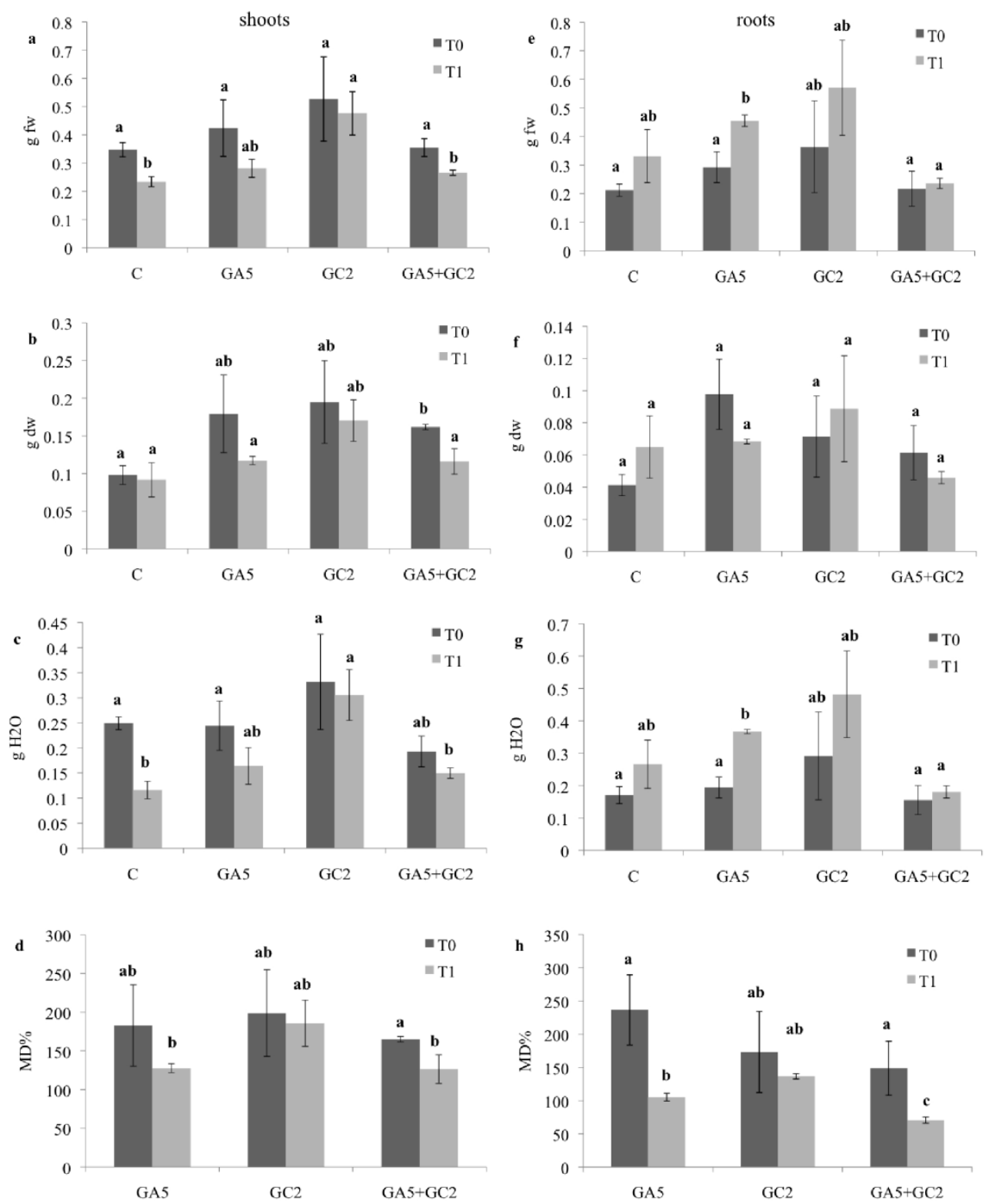

Figure 2. Pomegranate growing parameters in a-d shoots and e-h roots under sterile-soil transplant condition. Treatments: C (control without inoculation); GA5 (Rhizophagus intraradices GA5 strain inoculation); GC2 (Rhizophagus intraradices GC2 strain inoculation); GA5+GC2 (1:1 mixture of GA5 and GC2 strains). MD\% (mycorrhizal dependency percentage), fw (fresh weight), dw (dry weight), water content ( $\mathrm{g} \mathrm{H}_{2} \mathrm{O}$ ); T0 (on raised table); T1 (3 days transplanted on sterile soil). Different letters denote significant differences, P ( 0.05 . Data represent mean values \pm standard error of mean $(n=6)$. 
At T1, GA5-inoculated plants greatly increased CAT, SOD, and APX shoot enzyme activities (Figure 3a-c) without any changes in MDA content (Table 1). A significant decrease in PROT content was observed (Table 1). Under the same condition, GC2inoculated plants decreased CAT and SOD, although there were no significant differences in comparison to control plants. In addition, a significant increase in APX activity was observed (Figure 3a, c). However, the MDA content significantly increased, but these values were significantly lower than control plants under the same condition. Moreover, PROT was significantly higher at $\mathrm{T} 1$ but no differences were observed in comparison to control plants (Table 1). All enzymatic activities (Figure 3a-c) and PROT content in the shoots of co-inoculated plants were similar to the control treatment (Table 1) and only the MDA content was significantly lower than the control plants (Table 1).
Regarding roots, plants at $\mathrm{T} 0$ and inoculated with GA5 increased significantly SOD and APX enzyme activities (Figure 3e, f). However, MDA and PROT contents were similar to control plants (Table 1). Under T1, SOD and APX decreased at similar values to those of control plants (Figure 3e, f) and only CAT showed lower activity than T0. Moreover, CAT enzymatic activity was significantly higher than control plants under $\mathrm{T} 1$ condition (Figure 3d). The GC2 and co-inoculated plants had similar CAT activities, and increased APX compared to control plants (Figure 3d, f). For both samplings, SOD was similar in co-inoculated roots in comparison to control plants at T0. Only GC2-inoculated plants increased SOD activity (Figure 3e). Furthermore, the MDA and PROT contents were significantly higher in T0 compared to control plants, and particularly at T1, the MDA decreased to values similar to control plants at $\mathrm{T} 0$, and PROT increased in co-inoculated plants meanwhile no changes were observed in GC2 (Table 1). 

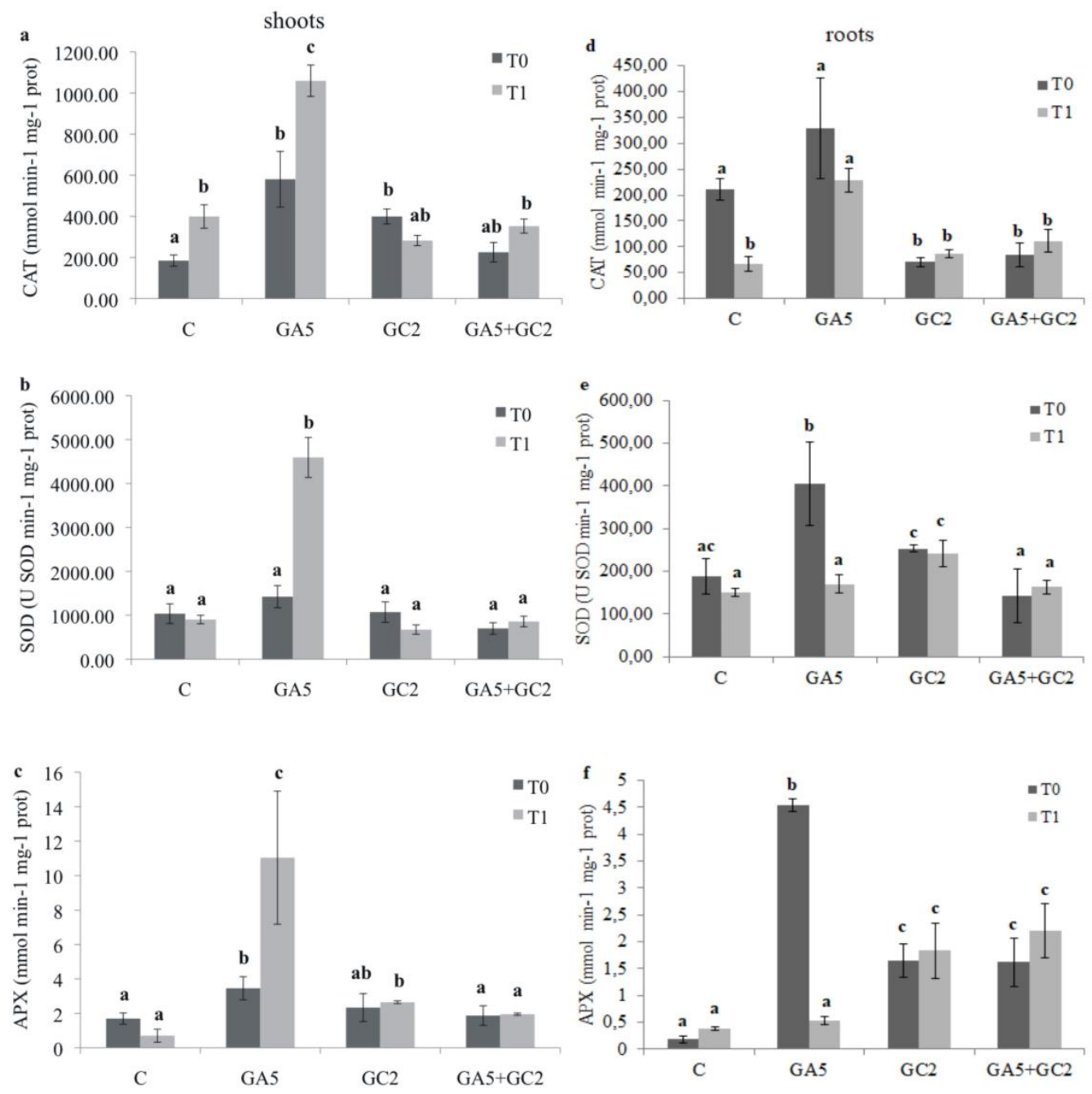

Figure 3. Enzyme activities: catalase (CAT); superoxide dismutase (SOD) and ascorbate peroxidase (APX) in pomegranate a-c shoots and d-f roots under sterile-transplant condition. Treatments: C (control without inoculation); GA5 (Rhizophagus intraradices GA5 strain inoculation); GC2 (Rhizophagus intraradices GC2 strain inoculation); GA5+GC2 (1:1 mixture of GA5 and GC2 strains). T0 (on raised table); T1 (3 days transplanted on sterile soil). Different letters denote significant differences, $\mathrm{P}$ ( 0.05 . Data represent mean values \pm standard error of mean $(n=6)$. 
Table 1. Protein (PROT) and Malon dihaldehyde (MDA) contents in pomegranate shoots and roots under steriletransplant condition.

\begin{tabular}{|c|c|c|c|c|}
\hline \multirow[t]{2}{*}{ Treatment } & \multicolumn{2}{|c|}{ PROT (mg ml-1) } & \multicolumn{2}{|c|}{ MDA (mg prot $\left.{ }^{-1}\right)$} \\
\hline & shoots & roots & shoots & roots \\
\hline \multicolumn{5}{|l|}{ T0 } \\
\hline C & $0.06 \pm 0.0001 \mathrm{a}$ & $0.03 \pm 0.0001 \mathrm{a}$ & $0.65 \pm 0.004 a$ & $0.16 \pm 0.009 a$ \\
\hline GA5 & $0.03 \pm 0.005 b$ & $0.03 \pm 0.001 \mathrm{a}$ & $0.70 \pm 0.01 \mathrm{a}$ & $0.12 \pm 0.001 \mathrm{a}$ \\
\hline $\mathrm{GC2}$ & $0.04 \pm 0.005 b$ & $0.04 \pm 0.0008 c$ & $0.51 \pm 0.006 c$ & $0.29 \pm 0.06 c$ \\
\hline $\mathrm{GA} 5+\mathrm{GC} 2$ & $0.07 \pm 0.0009 \mathrm{~d}$ & $0.04 \pm 0.001 c$ & $0.52 \pm 0.006 c$ & $0.28 \pm 0.01 \mathrm{c}$ \\
\hline \multicolumn{5}{|l|}{$\mathrm{T} 1$} \\
\hline C & $0.05 \pm 0.007 a b$ & $0.06 \pm 0.004 b$ & $0.93 \pm 0.01 b$ & $0.01 \pm 0.003 b$ \\
\hline GA5 & $0.01 \pm 0.0001 \mathrm{c}$ & $0.06 \pm 0.0001 b$ & $0.67 \pm 0.01 a$ & $0.02 \pm 0.0001 b$ \\
\hline GC2 & $0.06 \pm 0.001 \mathrm{a}$ & $0.04 \pm 0.002 \mathrm{c}$ & $0.65 \pm 0.03 a$ & $0.10 \pm 0.01 \mathrm{a}$ \\
\hline $\mathrm{GA} 5+\mathrm{GC} 2$ & $0.06 \pm 0.007 a$ & $0.06 \pm 0.001 b$ & $0.70 \pm 0.02 \mathrm{a}$ & $0.11 \pm 0.009 \mathrm{a}$ \\
\hline \multicolumn{5}{|l|}{ ANOVA } \\
\hline $\mathrm{T}$ & n.s. & $* * *$ & $* * *$ & $* * *$ \\
\hline AMF & $* * *$ & $* *$ & $* * *$ & $* * *$ \\
\hline $\mathrm{T} \times \mathrm{AMF}$ & $* *$ & $* * *$ & $* * *$ & n.s. \\
\hline
\end{tabular}

Treatments: C (control without inoculation); GA5 (Rhizophagus intraradices GA5 strain inoculation); GC2 (Rhizophagus intraradices GC2 strain inoculation); GA5+GC2 (1:1 mixture of GA5 and GC2 strains). T0 (on raised table); T1 (3 days transplanted on sterile soil). Different letters into each column denote significant differences, ${ }^{*} \mathrm{P}(0.05, * * \mathrm{P}(0.01, * * * \mathrm{P}(0.001$, not significant (n.s.). Data represent mean values \pm standard error of mean $(n=6)$. 
Twelve months after pomegranate cultivation under nursery conditions, a stabilization of AMF colonization was observed, maintaining a low mycorrhization and arbuscule percentages in GC2-inoculated and co-inoculated plants without changes in vesicles percentage (data not shown). However, all plants had survived until the end of the experiment (data not shown). Shoot fresh weight and water content were not affected by treatments nor transplant, but an increase in shoot biomass by transplant for all treatments was observed (Figure 4a, c). In addition, at T3, co-inoculated plants showed less MD followed by GC2-inoculated plants than GA5inoculated plants (Figure 4d). In roots, co-inoculated plants diminished in fresh weight in a similar manner in GC2-inoculated plants in contrast to control treatment (Figure 4e). Co-inoculation decreased root biomass at
T3 and a single inoculation showed no change under transplant condition, but was significantly lower than control plants at both conditions (Figure 4f). The transplant condition decreased root water content in inoculated treatments in contrast to control treatment. Moreover, this was markedly observed in GC2 and co-inoculated roots (Figure 4g). However, only coinoculated roots showed the highest MD at T2; however, at T3, they decreased (Figure 4h). Single inoculated plants showed a higher shoot: root ratio in $\mathrm{T} 2$, and $\mathrm{T} 3$ compared with control plants. Co-inoculation and control plants showed similar values at $\mathrm{T} 2$ but at T3, only co-inoculated plants demonstrated improved shoot: root ratio at similar values compared to single inoculation (Figure 5).

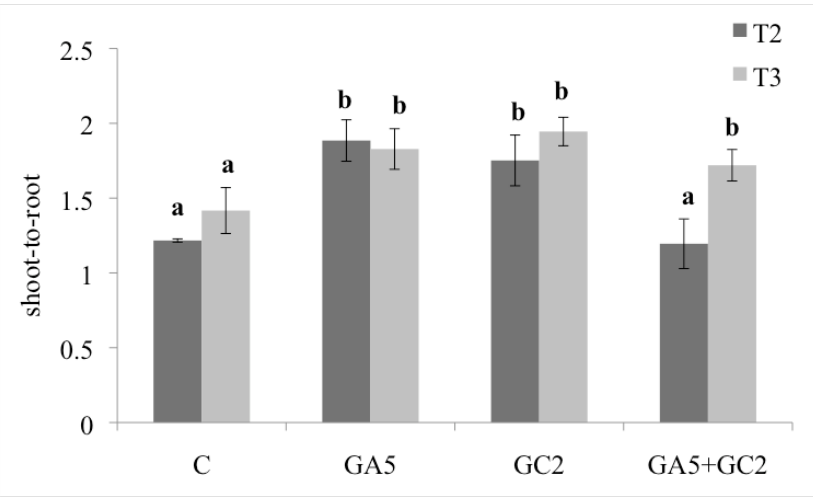

Figure 5. Shoot-to-root ratio in pomegranate plants under non-sterile transplant condition. Treatments: C (control without inoculation); GA5 (Rhizophagus intraradices GA5 strain inoculation); GC2 (Rhizophagus intraradices GC2 strain inoculation); GA5+GC2 (1:1 mixture of GA5 and GC2 strains). T2 (one year plants on sterile soil); T3 (7 days after transplant to non-sterile soil). Different letters denote significant differences, $\mathrm{P}(0.05$. Data represent mean values \pm standard error of mean $(n=6)$ 

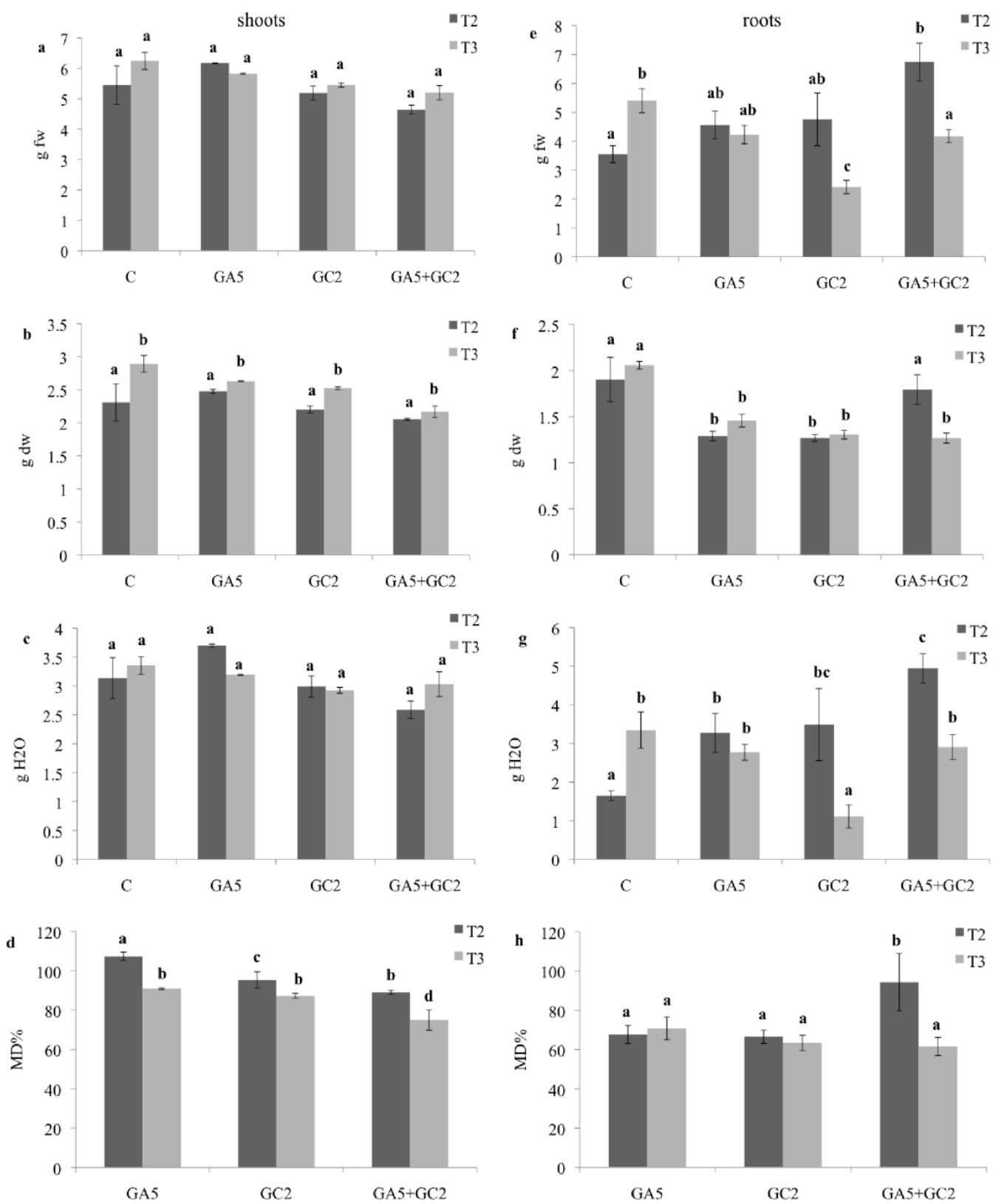

Figure 4. Pomegranate growing parameters in a-d shoots and e-h roots under non-sterile soil transplant condition. Treatments: C (control without inoculation); GA5 (Rhizophagus intraradices GA5 strain inoculation); GC2 (Rhizophagus intraradices GC2 strain inoculation); GA5+GC2 (1:1 mixture of GA5 and GC2 strains). MD\% (mycorrhizal dependency percentage), fw (fresh weight), dw (dry weight), water content $\left(\mathrm{g} \mathrm{H}_{2} \mathrm{O}\right)$; T2 (one year plants on sterile soil); T3 (7 days after transplant to non-sterile soil). Different letters denote significant differences, $\mathrm{P}$ ( 0.05. Data represent mean values \pm standard error of mean $(n=6)$. 

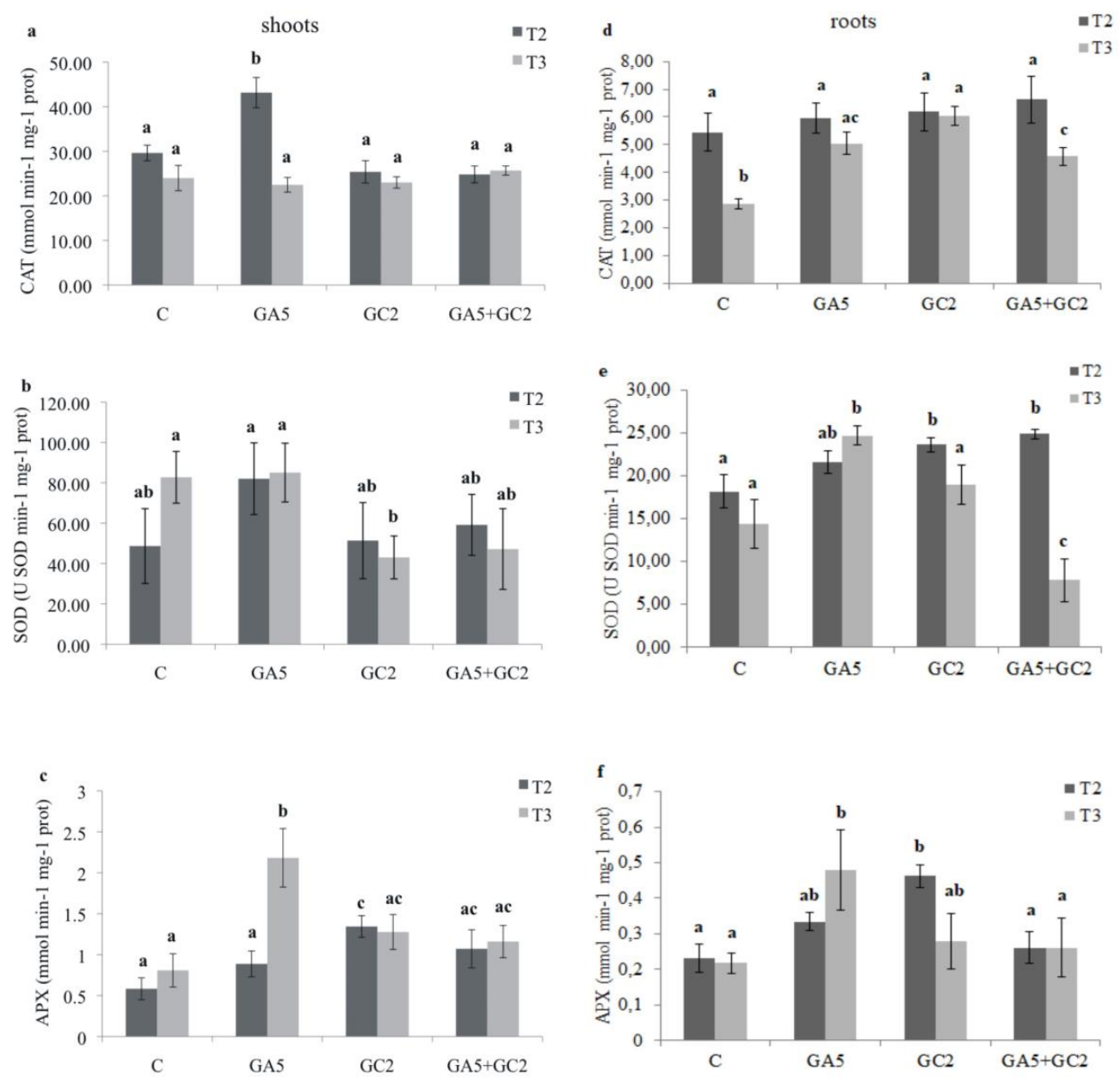

Figure 6. Enzyme activities: catalase (CAT); superoxide dismutase (SOD) and ascorbate peroxidase (APX) in pomegranate a-c shoots and d-f roots under non-sterile transplant condition. Treatments: $\mathrm{C}$ (control without inoculation); GA5 (Rhizophagus intraradices GA5 strain inoculation); GC2 (Rhizophagus intraradices GC2 strain inoculation); GA5+GC2 (1:1 mixture of GA5 and GC2 strains). T2 (one year plants on sterile soil); T3 (7 days after transplant to non-sterile soil). Different letters denote significant differences, P ( 0.05 . Data represent mean values \pm standard error of mean $(n=6)$. 
Table 2. Protein (PROT) and Malon dihaldehyde (MDA) contents in pomegranate shoots and roots under nonsterile-transplant condition.

\begin{tabular}{|c|c|c|c|c|}
\hline Treatment & \multicolumn{2}{|c|}{ PROT } & \multicolumn{2}{|c|}{ MDA } \\
\hline \multirow[b]{2}{*}{$\mathrm{T} 2$} & shoots & roots & shoots & roots \\
\hline & & & & \\
\hline C & $0.79 \pm 0.20 \mathrm{ac}$ & $1.31 \pm 0.001 \mathrm{a}$ & $0.64 \pm 0.003 a$ & $0.21 \pm 0.01 a$ \\
\hline GA5 & $0.59 \pm 0.002 a b$ & $1.30 \pm 0.003 \mathrm{a}$ & $1.28 \pm 0.02 c$ & $0.16 \pm 0.002 b$ \\
\hline GC2 & $0.59 \pm 0.001 \mathrm{a}$ & $1.31 \pm 0.002 \mathrm{a}$ & $0.74 \pm 0.008 d$ & $0.19 \pm 0.04 a b$ \\
\hline $\mathrm{GA} 5+\mathrm{GC} 2$ & $0.59 \pm 0.001 \mathrm{a}$ & $1.30 \pm 0.002 \mathrm{a}$ & $0.59 \pm 0.006 a$ & $0.21 \pm 0.009 a$ \\
\hline \multicolumn{5}{|l|}{ T3 } \\
\hline C & $0.60 \pm 0.001 \mathrm{ac}$ & $1.30 \pm 0.003 a$ & $0.50 \pm 0.003 b$ & $0.15 \pm 0.008 b$ \\
\hline GA5 & $0.59 \pm 0.0006 b$ & $1.31 \pm 0.001 \mathrm{a}$ & $0.63 \pm 0.01 \mathrm{a}$ & $0.14 \pm 0.002 b$ \\
\hline GC2 & $0.59 \pm 0.001 \mathrm{a}$ & $1.30 \pm 0.0005 a$ & $0.54 \pm 0.006 b$ & $0.21 \pm 0.04 a b$ \\
\hline GA5+GC2 & $0.60 \pm 0.0009 c$ & $1.30 \pm 0.0009 a$ & $0.61 \pm 0.008 a$ & $0.21 \pm 0.009 \mathrm{a}$ \\
\hline \multicolumn{5}{|l|}{ ANOVA } \\
\hline $\mathrm{T}$ & $*$ & n.s. & $* * *$ & $*$ \\
\hline AMF & $*$ & n.s. & $* * *$ & n.s. \\
\hline $\mathrm{T} \times \mathrm{AMF}$ & n.s. & n.s. & $* * *$ & n.s. \\
\hline
\end{tabular}

Treatments: C (control without inoculation); GA5 (Rhizophagus intraradices GA5 strain inoculation); GC2 (Rhizophagus intraradices GC2 strain inoculation); GA5+GC2 (1:1 mixture of GA5 and GC2 strains). T2 (one year plants on sterile soil); T3 (7 d after transplant to non-sterile soil). Different letters into each column denote significant differences, ${ }^{*} \mathrm{P}\left(0.05,{ }^{*} \mathrm{P}\left(0.01,{ }^{*} * * \mathrm{P}(0.001\right.\right.$, not significant (n.s.). Data represent mean values \pm standard error of mean $(n=6)$. 
In roots, GA5-inoculated plants increased CAT, SOD and APX enzymatic activities under T3 condition compared to control plants under the same condition (Figure 6d, f). A significant increase in SOD and APX were observed in GC2-inoculated plants at $\mathrm{T} 2$ and then decreased to values similar to control plants at T3 (Figure 6e, f). No changes were observed in CAT activity at $\mathrm{T} 2$ and $\mathrm{T} 3$, although these values were significantly higher than control plants at T3 (Figure 6d). Co-inoculation decreased CAT enzyme activity at T3. Moreover, these values were significantly higher than control plants (Figure 6d). However, SOD dramatically decreased in T3 (Figure 6e). There were no changes in MDA content, although they were significantly higher than control plants (Table 2). No differences were observed in PROT content for all treatments in T2 and T3 (Table 2).

\section{Discussion}

Plant survival evaluation plays an important role in transplantation acclimation. Alguacil et al. (2003) found high survival rates in non-mycorrhizal plants followed by the mixture of native AMF applied to three forest plants. Thus, despite demonstrating less survival in single inoculation, water content was higher particularly in the shoots. In roots, GA5-inoculated plants enhanced the water content. In addition, coinoculation showed no additional benefit at the initial growth stage. The mycorrhizal dependency parameter is useful to test the degree of plant maximum growth production under the mycorrhizal condition (Menge et al., 1978). In our experiments, at the initial stage of pomegranate cuttings growth, we found no evidence in mycorrhizal dependency to the transplantation protection condition. Nursery-controlled conditions combined with sterilized substrate could be the reason underlying the lack of any observed differences.
The protection against oxidative stress caused by the transplant was improved by GA5 strain an increase in more than one enzymatic activity and diminishing lipid damage. Previous work demonstrated that the GC2 strain improved pomegranate plants against oxidative stress at reduced water availability (Bompadre et al., 2014). The GC2 strain has a low growth rate observed in our laboratory compared to GA5 behavior (Silvani et al., 2014). The protective effects observed in pomegranate plants inoculated with the GC2 strain is delayed compared to the GA5 strain, which has an aggressive behavior. Transplant stress requires an early response and is the reason that the GA5 strain was more efficient in this experiment. Ascorbate peroxidase and SOD enzymes are important to detoxify $\mathrm{H}_{2} \mathrm{O}_{2}$ in chloroplasts, and thus plants are photosynthetically more active, thereby improving plant growth. Several studies have found an increase in the photosynthetic rate in mycorrhizal plants (Aseri et al., 2008; Singh et al., 2012), and thus, the photosynthetic apparatus in GA5 inoculated pomegranate was protected against oxidative stress. In particular, in GC2-inoculated plants, only APX was enhanced. Zou et al. (2014) suggested that the presence of AMF could induce antioxidant enzymes activities to prevent an over-accumulation of ROS as a protective mechanism. However, co-inoculation had no protective effect as demonstrated in the early growth stage. Studies performed in Retama sphaerocarpa under drought showed that phosphorus addition increased APX enzymatic activity in comparison to autochthonous AMF or $R$. intraradices inoculation (Armada et al., 2016). Alguacil et al. (2003) found that the effects of the mycorrhizal inoculation treatments on antioxidant enzyme activities varied depending on the shrub species. In particular, they observed a small increase in antioxidant enzyme activities in Olea europaea L. shoots. However, Wu et al. (2007) observed an ability to improve the water relations of Citrus tangerine 
Hort. ex Tanaka and five AMF tested and antioxidant enzyme activities.

In roots, the response to oxidative stress in sterile transplant soil was less evident on inoculated plants most likely due to better osmotic adjustment. Consistent with Mahmood and Rizvi (2010), AMF has the ability to promote plant survival in response to transplant stress.

During 12 months of pomegranate nursery growth, co-inoculation of plants showed fewer intraradical structures that may be due to either one of two fungal strains, which could be formed in the presence of each other, causing a competitive effect between symbionts. The same behavior was observed by Alkan et al. (2006), García et al. (2017), Janoušková et al. (2009) and Miranda et al. (2011). Moreover, host plants are preferentially colonized by specific strains of AMF (Zhu et al., 2000). It is known that mycorrhizal dependency can be altered by many variables in a given plant (Menge et al., 1978; Wu et al., 2007). Moreover, Alguacil et al. (2003) observed a synergistic effect in plants inoculated with a mixture of native AMF. In our experiment, a decrease in MD in the shoots was observed with non-sterile soil transplantation. Under these conditions, plants have to adapt to a new rhizospheric condition. Shoots of the inoculated plants were not affected by non-sterile transplant condition since the growth parameters were similar to control plants. However, GA5-inoculated plants had a higher MD. Moreover, root biomass was negatively affected and thus, inoculated plants had a higher shoot: root ratio. Protection mediated by antioxidative enzymes in shoots were negatively affected by non-sterile transplant condition particularly those inoculated with GC2 strain. Research studies concerning Lactuca sativa plants inoculated with a combination of selenobacteria and $R$. intraradices showed a synergistic protection against antioxidative stress (Durán et al., 2016). RuizLozano et al. (2012) stated that mycorrhizal plants demonstrate enhanced activity of several antioxidant enzymes, but the response of an individual enzyme has been shown to vary with respect to the fungal species and host plant. In our experiments, root tissue was actively defended mainly in GA5-inoculated plants without lipid damage.

\section{Conclusions}

In conclusion, our experiments demonstrated that early mycorrhizal inoculation in the plant production of Punica granatum L. generates healthy plants that are more protected against environmental stress. Pomegranate transplant stress tolerance improvement was particularly mediated by the GA5 strain, making the symbiotic AMF potentially useful in plant production. In addition, in comparison to previous work, we demonstrated that it is important to evaluate mycorrhizalhost combination depending of the final condition in which the plant will be established, to ensure optimization of the chosen combination.

\section{Acknowledgments}

The authors thank Consejo Nacional de Investigaciones Científicas y Técnicas (CONICET), Agencia Nacional de Promoción Científica y Tecnológica (ANCyPT) and Universidad de Buenos Aires (UBA) for financial support.

\section{References}

Aebi, H. 1984. Catalase in vitro. Methods Enzymol. 105:121-126.

Alguacil, M.M., Hernandez, J.A., Caravaca, F., Portillo, B., Roldan, A. 2003. Antioxidant enzyme activities in shoots from three mycorrhizal shrub species afforested in a degraded semi-arid soil. Physiol. Plant. 118: 562-570. 
Alkan, N., Gadkar, V., Yarden, Q., Kapulnik, Y. 2006. Analysis of quantitative interactions between two species of arbuscular mycorrhizal fungi, Glomus mosseae and $G$. intraradices, by real-time PCR. Appl. Environ. Microbiol. 72: 4192-4199.

Armada, E., López-Castillo, O., Roldán, A., Azcón, R. 2016. Potential of mycorrhizal inocula to improve growth, nutrition and enzymatic activities in retama sphaerocarpa compared with chemical fertilization under drought conditions. J. Soil Sci. Plant Nutr. 16: 380-399.

Aseri, G., Jain, N., Panwar, J., Rao, A., Meghwal, P. 2008. Biofertilizers improve plant growth, fruit yield, nutrition, metabolism and rhizosphere enzyme activities of Pomegranate (Punica granatum L.) in Indian Thar Desert. Sci. Hortic. (Amsterdam). 117: 130-135.

Beyer, W.F., Fridovich, I. 1987. Assaying for superoxide dismutase activity: Some large consequences of minor changes in conditions. Anal. Biochem. 161: 559-566.

Bompadre, M.J., Silvani, V.A., Fernández Bidondo, L., Ríos de Molina, M.D.C., Colombo, R.P., Pardo, A.G., Godeas, A.M. 2014. Arbuscular mycorrhizal fungi alleviate oxidative stress in pomegranate plants growing under different irrigation conditions. Bot. 92: 187-193.

Bradford, M.M. 1976. A rapid and sensitive method for the quantitation of microgram quantities of protein utilizing the principle of protein-dye binding. Anal. Biochem. 72: 248-254.

Clewer, A.G., Scarisbrick, D. 2001. Practical statistics and experimental design for plant and crop science. Factorial Exp.: John Wiley and Sons Ltd (ed.), Factorial Exp.pp. 159-181. West Sussex England.

Durán, P., Acuña, J., Armada, E., López-Castillo, O., Cornejo, P., Mora, M., Azcón, R. 2016. Inoculation with selenobacteria and arbuscular mycor- rhizal fungi to enhance selenium content in lettuce plants and improve tolerance against drought stress. J. Soil Sci. Plant Nutr. 16: 211-225.

Franck, N. 2009. Producción y manejo de plantaciones de granado en Chile, Israel y Argentina. Granados, Perspectivas y oportunidades un Negocio Emergente: C. Castillo (ed.), pp. 28-35. Chile: Fundación Chile.

García, S., Pezzani, F., Rodríguez-Blanco, A. 2017. Long-term phosphorus fertilization effects on arbuscular mycorrhizal fungal diversity in Urugayan grasses. J. Soil Sci. Plant Nutr. 17 (4): 1013-1027.

Helgason, T., Merryweather, J.W., Denison, J., Wilson, P., Young, J.P.W., Fitter, A.H. 2002. Selectivity and functional diversity in arbuscular mycorrhizas of co-occurring fungi and plants from a temperate deciduous woodland. J. Ecol. 90: 371-384.

Hewitt, E. 1952. Sand and water culture methods in the study of plant nutrition. Tech. Commun. Commonw. Bur. Hortic. Plant. Crop. 241.

Hodges, D.M., Delong, J.M., Forney, C.F., Prange, R.K. 1999. Improving the thiobarbituric acid-reactive-substances assay for estimating lipid peroxidation in plant tissues containing anthocyanin and other interfering compounds. Planta. 207: 604-611.

Hossain, M.A., Asada, K. 1984. Inactivation of ascorbate peroxidase in spinach chloroplasts on dark addition of hydrogen peroxide: its protection by ascorbate. Plant Cell Physiol. 25: 1285-1295.

Janoušková, M., Seddas, P., Mrnka, L., van Tuinen, D., Dvořáčková, A., Tollot, M., Gianinazzi-Pearson, V, Vosátka M, Gollotte, A. 2009. Development and activity of Glomus intraradices as affected by co-existence with Glomus claroideum in one root system. Mycorrhiza. 19: 393-402. 
Khattab, M.M., Shaban, A.E., El-shrief, A.H., Mohamed, A.S.E. 2011. Growth and productivity of pomegranate trees under different irrigation levels. III: leaf pigments, proline and mineral content. J. Hortic. Sci. Ornam. Plants. 3: 265-269.

Knight, P.R., Coker, C.H., Anderson, J.M., Murchison, D.S., Watson, C.E. 2005. Mist interval and K-IBA concentration influence rooting of Orange and Mountain azalea. Nativ. Plants. 6: 111-117.

Koller, G. 1977. Transplanting stress - a view from the plant's perspective. Arnoldia. 37: 230-241.

Liptay, A., Sikkema, P., Fonteno, W. 1998. Transplant growth control through water deficit stress-A review. HortTechnology. 8: 540-543.

Mahmood, I., Rizvi, R. 2010. Mycorrhiza and organic farming. Asian J. Plant Sci. 9: 241-248.

Marin, M. 2005. Arbuscular mycorrhizal inoculation in nursery practice. Handbook of Microbial Biofertilizers: M. Rai (ed.), 289-324. New York. London. Oxford: Food Products Press ${ }^{\circledR}$.

McGonigle, T.P., Miller, M.H., Evans, D.G., Fairchild, G.L., Swan, J.A. 1990. A new method which gives an objective measure of colonization of roots by vesicular- arbuscular mycorrhizal fungi. New Phytol. 115: 495-501.

Menge, J., Johnson, E., Platt, R. 1978. Mycorrhizal dependency of several citrus cultivars under three nutrient regimes. New Phytol. 81: 553-559.

Miranda, D., Fischer, G., Ulrichs, C. 2011. The influence of arbuscular mycorrhizal colonization on the growth parameters of cape gooseberry (Physalis peruviana $\mathrm{L}$.) plants grown in a saline soil. J. Soil Sci. Plant Nutr. 11: 18-31

Mittler, R., Vanderauwera, S., Gollery, M., Van Breusegem, F. 2004. Reactive oxygen gene network of plants. Trends Plant Sci. 19: 393-402.
Munkvold, L., Kjøller, R., Vestberg, M., Rosendahl, S., Jakobsen, I. 2004. High functional diversity within species of arbuscular mycorrhizal fungi. New Phytol. 164: 357-364.

Parodi, L. 1978. Enciclopedia Argentina de Agricultura y Jardinería. (ACME, Ed.). Buenos Aires.

Ruiz-Lozano, J.M. 2003. Arbuscular mycorrhizal symbiosis and alleviation of osmotic stress. New perspectives for molecular studies. Mycorrhiza, 13: 309-17.

Ruiz-Lozano, J.M., Porcel, R., Azcón, C., Aroca, R. 2012. Regulation by arbuscular mycorrhizae of the integrated physiological response to salinity in plants: New challenges in physiological and molecular studies. J. Exp. Bot. 63: 4033-4044.

Silvani, V.A., Fernández Bidondo, L., Bompadre, M.J., Colombo, R.P., Pérgola, M., Bompadre, A., Fracchia, S., Godeas, A. 2014. Growth dynamics of geographically different arbuscular mycorrhizal fungal isolates belonging to the "Rhizophagus clade" under monoxenic conditions. Mycologia. 106: 963-975.

Singh, N., Singh, S., Singh, A., Meshram, D., Suroshe, S., Mishra, D. 2012. Arbuscular mycorrhizal fungi (AMF) induced hardening of micropropagated pomegranate (Punica granatum L.) plantlets. Sci. Hortic. (Amsterdam). 136: 122-127.

Violi, H.A., Treseder, K.K., Menge, J.A., Wright, S.F., Lovatt, C.J. 2007. Density dependence and interspecific interactions between arbuscular mycorrhizal fungi mediated plant growth, glomalin production, and sporulation. Can. J. Bot. 85: 63-75.

Wu, Q.S., Xia, R.X., Zou, Y.N. 2008. Improved soil structure and citrus growth after inoculation with three arbuscular mycorrhizal fungi under drought stress. Eur. J. Soil Biol. 44: 122-128.

Wu, Q.S., Zou, Y.N. 2009. Mycorrhiza has a direct effect on reactive oxygen metabolism of droughtstressed citrus. Plant, Soil Environ. 55: 436-442. 
Wu, Q.S., Zou, Y.N., Liu, W., Ye, X.F., Zai, H.F., Zhao, L.J. 2010. Alleviation of salt stress in citrus seedlings inoculated with mycorrhiza: Changes in leaf antioxidant defense systems. Plant, Soil Environ. 56: 470-475.

Wu, Q.S., Zou, Y.N., Xia, R.X. 2006. Effects of water stress and arbuscular mycorrhizal fungi on reactive oxygen metabolism and antioxidant production by citrus (Citrus tangerine) roots. Eur. J. Soil Biol. 42: 166-172.

Wu, Q.S., Zou, Y.N., Xia, R.X., Wang, M.Y. 2007. Five Glomus species affect water relations of Citrus tangerine during drought stress. Bot. Stud. 48: 147-154.
Zhu, X.C., Song, F.B., Liu, S.Q., Liu, T.D., Zhou, X. 2012. Arbuscular mycorrhizae improves photosynthesis and water status of Zea mays L. under drought stress. Plant Soil Environ. 58: 186-191.

Zhu, Y.G., Laidlaw, A., Christie, P., Hammond, M.E. 2000. The specificity of arbuscular mycorrhizal fungi in perennial ryegrass-white clover pasture. Agric. Ecosyst. Environ. 77: 211-218.

Zou, Y.N., Huang, Y.M., Wu, Q.S., He, X.H. 2014. Mycorrhiza-induced lower oxidative burst is related with higher antioxidant enzyme activities, net $\mathrm{H}_{2} \mathrm{O}_{2}$ effluxes, and $\mathrm{Ca}_{2}{ }^{+}$influxes in trifoliate orange roots under drought stress. Mycorrhiza. 25: $143-152$. 